'Departamento de Salud Pública, Universidad del Norte. Barranquilla, Colombia.

¿Universidad Simón Bolívar. Barranquilla, Colombia. ${ }^{3}$ Fundación Vida Nueva. Barranquilla, Colombia. ${ }^{4}$ Despacho del alcalde distrital de Barranquilla, Colombia.

${ }^{5}$ Departamento de matemática y estadística. Universidad del Norte. Barranquilla, Colombia.

anstrumentador quirúrgico. ${ }^{b}$ Economista. 'Matemática. dBiólogo.

Fuente de apoyo financiero: Fundación Mundial de Diabetes (WDF).

Los autores declaran no tener conflictos de interés.

Recibido el 27 de agosto de 2018, aceptado el 4 de marzo de 2019.

Correspondencia a: Víctor Flórez-García Departamento de Salud Pública, Universidad del Norte. Barranquilla, Colombia. Dirección: Km 5 Vía puerto Colombia. vfloreza@uninorte.edu.co

\section{Diabetes gestacional: implementación de una guía para su detección en la atención primaria de salud}

\author{
RAFAEL TUESCA MOLINA ${ }^{1}$, TANIA ACOSTA VERGARA ${ }^{1}$, \\ BRAYAN DOMÍNGUEZ LOZANO 2,a, CARLOS RICAURTE ${ }^{3, \mathrm{~b}}$, \\ HUMBERTO MENDOZA CHARRIS ${ }^{4}$, KAREN FLÓREZ-LOZANO ${ }^{5, c}$, \\ VÍCTOR FLÓREZ-GARCÍA ${ }^{1, \mathrm{~d}}$

\section{Implementation of a clinical guideline for detection of gestational diabetes in primary care}

Background: Gestational diabetes (GD) impacts maternal and fetal morbidity. In 2012, the World Diabetes Foundation provided financing project in Barranquilla (Colombia), aimed to implement a clinical guide for the diagnosis and treatment of this condition. Aim: To estimate the adherence of the guide in primary care centers. Material and Methods: A Cross-sectional study of pregnant women suspected of having gestational diabetes. Pregnant women were classified according to the week of admission to the prenatal control program: < week 24 or later. Women with a fasting blood glucose over $92 \mathrm{mg} / \mathrm{dL}$ and under $126 \mathrm{mg} / \mathrm{dL}$ or with some positive result to the oral glucose tolerance test were included. Results: Nine percent (1,887 women) of 21,699 registries of pregnant women, were at risk for gestational diabetes. Of these, 1,880 registries with complete data were analyzed. Sixty nine entered the program at less than 24 weeks of pregnancy and $71 \%$ had had a fasting blood glucose measured in the first control. In $69.2 \%$ of these women, criteria for gestational diabetes was met. A glucose tolerance test was suggested to women with a blood glucose below $92 \mathrm{mg} / \mathrm{dl}$. Among $72 \%$ of the latter, the glucose tolerance test met the criteria for gestational diabetes. Among the 498 women who entered the program after 24 weeks of gestation, $68 \%$ met the criteria for gestational diabetes with the fasting blood glucose levels. In 90 women, a glucose tolerance test was performed and $80 \%$ met the criteria for gestational diabetes. Conclusions: An adequate adherence to guidelines favors the detection of pregnant women with gestational diabetes.

(Rev Med Chile 2019; 147: 190-198)

Key words: Colombia; Diabetes, Gestational; Guideline; Mass Screening; Practice Guideline.
L a diabetes gestacional (DG) es el trastorno endocrino metabólico generado por una intolerancia a los hidratos de carbono que ocurre durante el embarazo, cuando los niveles de glicemia en ayunas son $\geq 92 \mathrm{mg} / \mathrm{dL}$ y/o al realizar la curva de tolerancia oral a la glucosa (solución glucosada de $75 \mathrm{mg}$ ) los resultados de la glicemia a la hora son $\geq 180 \mathrm{mg} / \mathrm{dL}$ y/o a las dos horas $\geq 153$ $\mathrm{mg} / \mathrm{dL}^{1-6}$. El efecto diabetógeno de esta entidad aumenta en la medida que avanza el embarazo, especialmente en el segundo trimestre, por acción de hormonas hiperglucemiantes, lo que supone un riesgo para la gestante y su producto. Este hecho es particularmente evidente si la enfermedad no 
es diagnosticada y controlada a tiempo, causando problemas de macrosomia fetal, fetopatía diabética con hiperinsulinismo fetal, hipoxia y acidosis fetal $^{2,3,7,8}$.

Las mujeres con DG presentan trastornos metabólicos similares a los ocurridos en personas diagnosticadas con diabetes tipo 2 (DT2) como la resistencia a la insulina y el consecuente daño celular' ${ }^{9}$. Después del parto, aun cuando se regresa a un nivel de glicemia normal, un alto porcentaje de estas mujeres desarrolla DT2 a lo largo de la vida $^{10-15}$.

La Organización Mundial de la Salud (OMS) considera que independientemente de su etiología, antigüedad y evolución posterior, la DG incluye diferentes circunstancias que la convierten en un riesgo y problema de salud pública, como son: la existencia de diabetes no conocida y descubierta durante la gestación o su aparición de novo, las complicaciones obstétricas y los problemas pre y postnatales ${ }^{14,15}$. Por lo anterior, y ante la disparidad de asumir criterios diagnósticos y la carga de enfermedad tanto para la gestante y en el futuro para ella y su producto, la OMS señala que el esfuerzo se debe orientar a acciones a nivel mundial para reducir la carga de la enfermedad e identificar actuaciones costo-eficientes para la salud materna e infantil. En este sentido, la OMS ha hecho un llamado a nivel global para que se impacte de manera positiva en las gestantes de riesgo en países de ingresos altos y bajos, dado que cerca a 20\%-30\% de ellas desarrollarán DG o se convertirán en diabéticas tipo 2 con posterioridad al embarazo, acompañándose de otras comorbilidades como hipertensión, dislipidemia y enfermedad cardiovascular ${ }^{1,14-16}$.

La incidencia de DG presenta variaciones a nivel mundial de $2 \%$ a $14 \%$, mientras que la prevalencia oscila de $2 \%$ a $70 \%$ a nivel global ${ }^{15,20-22}$. Las razones de la variabilidad en la prevalencia e incidencia se relacionan con aspectos de tipo étnico-racial, criterios diagnósticos de DG y prevalencia de diabetes tipo 2 en la población. Los criterios diagnósticos empleados hasta la fecha muestran diferencias con respecto al umbral de estimación en función del tamizaje a partir del riesgo de la gestante, la tipificación de prediabetes o intolerancia a los hidratos de carbono, DG, diabetes y la cantidad de sobrecarga de glucosa en las pruebas de cribado. Cuando se utiliza el criterio del Grupo Internacional de Estudio de Diabetes en Embarazadas (IADPSG) ${ }^{5}$, se incrementa la detección de DG, duplicándose o elevándose 8 veces su estimación y potenciando asociación con factores de riesgo de desenlace negativo en los productos o la gestación (preeclampsia, cesárea e incremento en la gestación). Si se compara con los criterios de la asociación canadiense de diabetes (CDA) en países con alta prevalencia se incrementa la clasificación, lo que se traduce en incrementar resultados perinatales adversos ${ }^{5,23}$. Sin embargo, al comparar con los criterios adoptados por la OMS en 1999 y 2013 con los de IADPSG en impactos perinatales no se evidencian variaciones importantes y se mantiene un ligero incremento en la detección ${ }^{5,9,11}$.

En la ciudad de Barranquilla, Colombia, se desconocía la prevalencia de esta condición en las gestantes. No existían en su momento criterios unificados o adopción de consenso para clasificar las embarazadas y protocolizar una guía de detección de la DG e intervención. Ante esta coyuntura, la Fundación Mundial de Diabetes (WDF) financió un proyecto denominado "Vida Nueva", que involucró a instituciones académicas de la ciudad, la Secretaría distrital de salud de Barranquilla y la Institución Prestadora de Servicios de Salud del régimen público. Esta alianza, favoreció la construcción de una guía de detección y atención para la DG al igual que la adopción de la misma durante la fase del estudio desde el año 2012 al $2014^{24}$.

La evaluación de la implementación de esta guía, permitirá identificar aspectos fuertes y débiles de la ruta establecida para la detección de DG por parte de los profesionales de la salud del primer nivel de la red pública de la ciudad. El objetivo de este estudio fue estimar la adherencia en la adopción de la guía para el tamizaje de diabetes gestacional en los centros de atención primaria de la ciudad de Barranquilla.

\section{Materiales y Métodos}

Se diseñó un estudio observacional de corte trasversal, a partir de la base de datos del servicio prestador de salud de las gestantes en riesgo que acudieron a los centros de atención primaria de la red pública para control prenatal desde enero de 2012 a diciembre de 2014 en la ciudad de Barranquilla, Colombia. Las unidades de análisis fueron los casos con alto riesgo de padecer DG de acuerdo con los criterios de clasificación del 
riesgo adoptados por la guía, considerándose en alto riesgo aquellas embarazadas que presentan uno o más de los siguientes factores ${ }^{25,26}$ : -edad $>25$ años, obesidad (IMC $>30 \mathrm{~kg} / \mathrm{m}^{2}$ ) o aumento del $110 \%$ del peso ideal -macrosomía en partos anteriores $(>4 \mathrm{~kg})$, antecedentes personales de DG, antecedentes de DM en familiares de primer grado, historia personal de tolerancia anormal de la glucosa, conocimiento de padecer de síndrome de ovarios poliquístico, hipertensión esencial o relacionada con el embarazo, uso de esteroides, peso materno al nacer : $>4,1 \mathrm{~kg}$ y/o 9 libras o $<$ de
2,7 kg y/o 6 libras, excesivo aumento de peso en el II y III trimestre.

Las gestantes fueron clasificadas en 2 grupos de acuerdo con el tiempo de gestación en el momento de su ingreso en el programa de control prenatal: Grupo 1, antes de o en la semana 24 de gestación y Grupo 2, posterior a la semana 24 de gestación.

\section{Descripción del algoritmo establecido en la Guía} para la detección de la DG (Figura 1)

El enfoque para el tamizaje fue el de "un paso a paso" propuesto por el Consenso de la

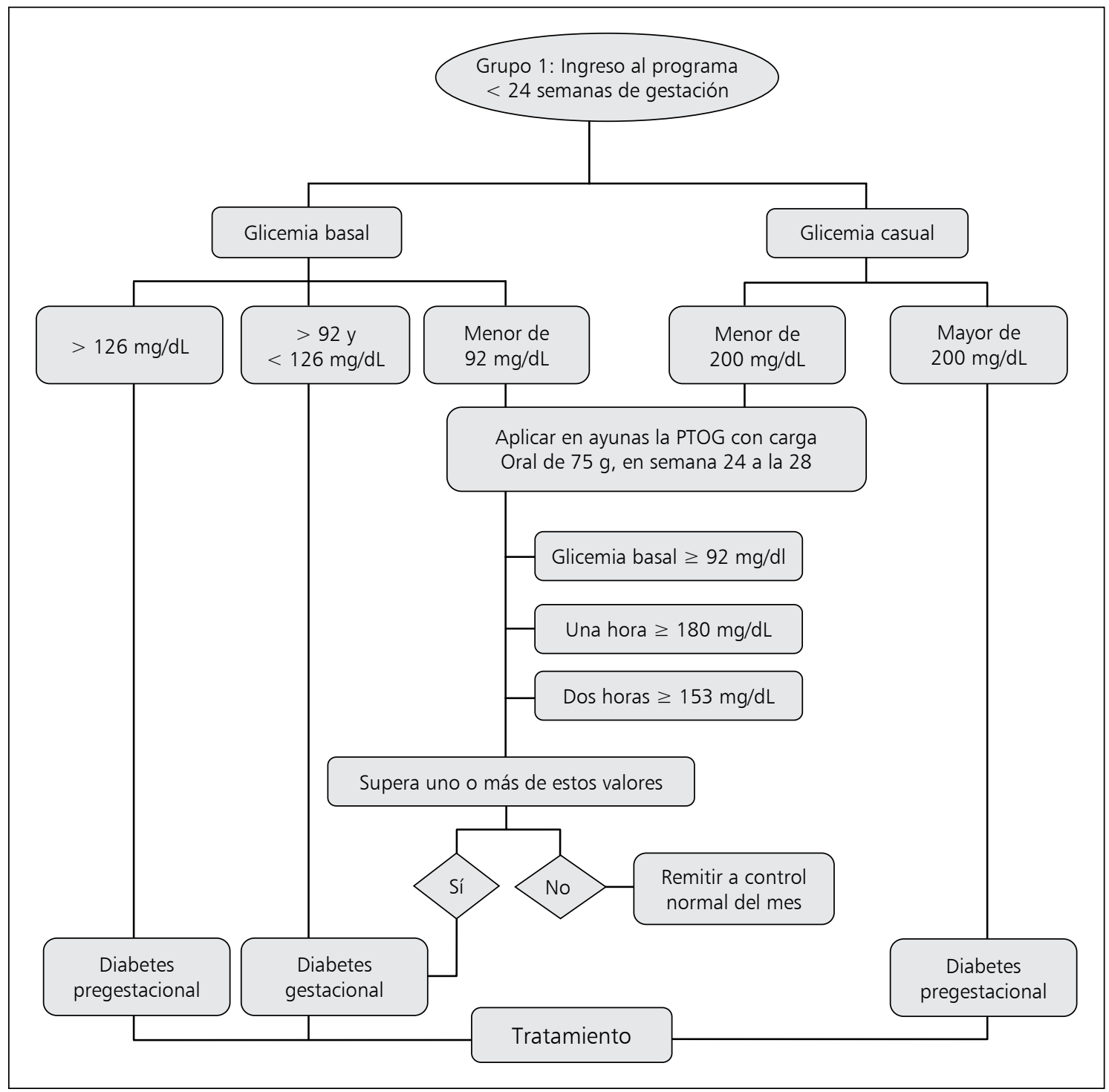

Figura 1. Algoritmo de manejo para gestantes con riesgo de Diabetes Gestacional, Barranquilla 2012-2014. 
IADPSG. El tamizaje se realizó en la $1^{\text {a }}$ visita de control del embarazo mediante glicemia basal o glicemia casual para hacer la inmediata valoración y detectar diabetes pre-gestacional (DGP) o DG. Se recomienda que la gestante se haga el primer control prenatal entre las semanas 7 a la 12 de embarazo. Teniendo el resultado de la glicemia basal de primera oportunidad (GB) se clasificó a la gestantes en una de tres categorías; 1) si el resultado de la glicemia es mayor o igual a 126 $\mathrm{mg} / \mathrm{dL}$ es catalogada como DPG y la gestante sería remitida siguiendo la ruta del algoritmo a médico especialista para su tratamiento; 2) si el resultado es mayor o igual a $92 \mathrm{mg} / \mathrm{dL}$ y menor de $126 \mathrm{mg} / \mathrm{dL}$ se cataloga como DG y sería remitida a especialista para su tratamiento teniendo en cuenta la ruta de la guía; 3) si el resultado de la glicemia basal es menor a $92 \mathrm{mg} / \mathrm{dL}$ se ordena la realización de la prueba de tolerancia oral a la glucosa (PTOG) de tres tiempos [glicemia basal, glicemia $1 \mathrm{~h}$ pos carga y glicemia a las $2 \mathrm{~h}$ pos carga] con carga de $75 \mathrm{~g}$ de solución glucosada entre la semana 24 a 28 de gestación. Durante la realización de la PTOG si la gestante presentaba 1 o más de los siguientes criterios alterados en su resultado era diagnosticada como DG (1 - glicemia basal mayor o igual a 92 $\mathrm{mg} / \mathrm{dL}$, 2- glicemia $1 \mathrm{~h}$ mayor o igual a $180 \mathrm{mg} / \mathrm{dL}$ y 3 - glicemia a las $2 \mathrm{~h}$ mayor o igual a $153 \mathrm{mg} / \mathrm{dL}$ ).

Fue calculado el promedio del porcentaje total de la adherencia de los profesionales a la aplicación y seguimiento de la guía de DG; se midió, además, la edad, semana de ingreso al programa de control prenatal que clasifica la población en dos grupos, realización de GB, realización de PTOG, cumplimiento de criterios para DG y su referencia al especialista para el tratamiento indicado.

El universo del estudio estuvo conformado por 21.699 registros de gestantes que acudieron a consulta a los 22 centros de salud de la ciudad, de los cuales 1.897 se clasificaron en gestantes de alto riesgo para DG, y a partir de esta se filtró la base de datos del proyecto "Vida Nueva". Los datos fueron depurados obteniéndose 1.880 registros. Cabe señalar que se presentan los resultados hasta la clasificación, no se evaluó tratamiento ordenado ni seguimiento.

Se estimaron proporciones de gestantes que cumplieron el algoritmo de acuerdo a los criterios de clasificación y pruebas solicitadas, dichas estimaciones estuvieron acompañadas de los respectivos intervalos de confianza al 95\%, para lo cual además se realizaron comparaciones de proporciones utilizando Chi-cuadrado con un nivel de significancia del 0,05. Se estimó además la prevalencia global de DG, período de detección, grupo y distribución por pruebas bioquímicas como medidas indirectas de la adherencia al protocolo. Para el análisis se empleó el paquete estadístico SPSS v. 23 en español. Esta investigación fue avalada por el Comité de Ética de la Universidad del Norte.

\section{Resultados}

Durante el primer y segundo año del estudio se observó un incremento estadísticamente significativo de 43,7 puntos porcentuales en la adopción del programa $(\mathrm{p}<0,00)$, este comportamiento ascendente pero no sostenido fue observado también para el tercer año $(\mathrm{p}<0,00)$. De acuerdo con los datos disponibles se estimó una proporción global de detección al tercer año de 8,7 de cada 100 embarazadas que acudieron a control prenatal por primera vez.

Se observó además que hubo una mayor adherencia entre las embarazadas que ingresaron al programa prenatal antes la semana 24 de gestación en comparación con las que ingresaron posterior a esta fecha $(\mathrm{p}<0,000)($ Tabla 1$)$.

El 69,41\% $(\mathrm{n}=1.305)$ de gestantes ingresaron a control prenatal antes de cumplir las 24 semanas de embarazo (Grupo 1). De estas, 71,9\% ( $\mathrm{n}=939)$ se realizaron la $\mathrm{GB}$, siendo diagnosticadas como DPG $2,9 \%(\mathrm{n}=28)$ y DG $69,2 \%$. A las gestantes con una estimación menor de $92 \mathrm{mg} / \mathrm{dL}$ en la GB, se les sugirió realizarse la PTGO, cumpliendo el procedimiento sugerido $80,5 \%$ del total de las cuales se detectaron $71,9 \%$ en la categoría de DG (Tabla 2, Figura 2).

El 26,5\% $(\mathrm{n}=498)$ de las gestantes ingresó al control prenatal después de la semana 24 de gestación (Grupo 2) del total de gestantes $68,1 \%$ de ellas $(n=216)$ fueron clasificadas de DG y a 90 se les solicitó realizar prueba de tolerancia oral a la glucosa detectándose en este grupo 80,2\% de DG (Figura 3, Tabla 2).

\section{Discusión}

La implementación de la guía permitió disponer de una estimación puntual de la prevalencia global de DG dentro del programa, muy similar 
Tabla 1. Características generales de las participantes en el estudio "Vida Nueva" del programa de riesgo de DG en la IPS Universitaria. Barranquilla 2012-2014

\begin{tabular}{|c|c|c|c|c|c|c|}
\hline & $\begin{array}{c}n^{*} \\
1.880\end{array}$ & $\begin{array}{c}\% \\
100\end{array}$ & & IC95\% & & $\mathbf{p}^{ \pm}$ \\
\hline \multicolumn{7}{|l|}{ Ingreso al programa (año) } \\
\hline 2012 & 21 & 1,12 & 0,6 & - & 1,6 & Ref \\
\hline 2013 & 842 & 44,79 & 42,5 & - & 47,1 & 0,000 \\
\hline 2014 & 1.017 & 54,1 & 51,8 & - & 56,4 & 0,000 \\
\hline Detección por primera vez $(n=21.609)$ & 1.880 & 8,7 & 8,3 & - & 9,00 & \\
\hline \multicolumn{7}{|l|}{ Grupo de detección } \\
\hline Grupo $1 * *$ & 1.305 & 69,41 & 67,3 & - & 71,5 & 0,000 \\
\hline Grupo 2*** & 498 & 26,49 & 24,4 & - & 28,5 & 0,000 \\
\hline Sin datos & 77 & 4,1 & 3,2 & - & 5,00 & \\
\hline
\end{tabular}

Fuente: Datos de vigilancia del estudio "Vida Nueva". * Población en riesgo de DG **Grupo 1: Ingreso al programa de control prenatal antes de la semana 24 de gestación; ${ }^{* * *}$ Grupo 2: Ingreso al programa de control prenatal después de la semana 24 de gestación. La clasificación de los grupos se hace de acuerdo a la guía. ${ }^{\ddagger}$ Chi cuadrado de Pearson.

Tabla 2. Adherencia a la guía Diagnóstico para la Diabetes Gestacional proyecto Vida Nueva en Barranquilla 2012-2014

\begin{tabular}{|c|c|c|}
\hline Total de Gestante en base de datos & $\begin{array}{l}n \\
1.880\end{array}$ & $\begin{array}{c}\% \\
100\end{array}$ \\
\hline \multicolumn{3}{|l|}{ Grupo 1: Ingreso al programa de control prenatal antes de la semana 24 de gestación [69,4\%] } \\
\hline Realización de Glicemia basal & 939 & 71,90 \\
\hline Gestantes con Glicemia $<92 \mathrm{mg} / \mathrm{dL}$ [ $\mathrm{n}=261$ ] que siguen el algoritmo y se les realiza la PTOG & 210 & 80,40 \\
\hline Gestantes diagnosticadas con DG que son referidas y siguen al tratamiento [ $=151]$ & 151 & 100,00 \\
\hline Promedio de cumplimiento* & & 84,10 \\
\hline \multicolumn{3}{|l|}{$\begin{array}{l}\text { Grupo 2: Ingreso al programa de control prenatal posterior a semana } 24 \text { de gestación semanas } \\
n=498[26,5 \%]\end{array}$} \\
\hline Realización de glicemia basal & 317 & 63 \\
\hline Gestante con glicemia normal < 92 mg/dL [n = 90]. Realización de PTOG & 76 & 84,5 \\
\hline Gestantes diagnosticada con DG que siguen al tratamiento [61] & 31 & 100,00 \\
\hline Promedio de cumplimiento* & & 82,50 \\
\hline
\end{tabular}

$p<0,05$.

con hallazgos reportados en otras latitudes ${ }^{5,24,26}$. Se observa un incremento en la detección de gestantes sospechosas de diabetes, dado que no se disponía antes de esta herramienta con criterios unificados en esta municipalidad para convertirse en un referente local y regional de gestantes sospechosas de DG. Esta estimación aporta una línea de base para el seguimiento del programa en la red pública de prestadores de salud del primer nivel.

Detectar de manera oportuna las gestantes sospechosas antes de la semana 24 y motivar al equipo de salud en la implementación del algoritmo y la derivación especializada a los puntos de atención de riesgo es uno de los logros de mayor importancia de este trabajo. Sin embargo, la evidencia disponible sugiere enfocar el tamizaje a partir de factores riesgo, tales como: multiparidad, edad mayor de 25 años en la gestante, antecedente de diabetes gestacional, de diabetes en línea familiar de primer grado, obesidad, síndrome de ovarios poliquísticos, hipertensión esencial o relacionada con el embarazo y uso de corticoides entre otros. No obstante, algunos autores sostienen que la diabetes gestacional se puede presentar en el tercer 


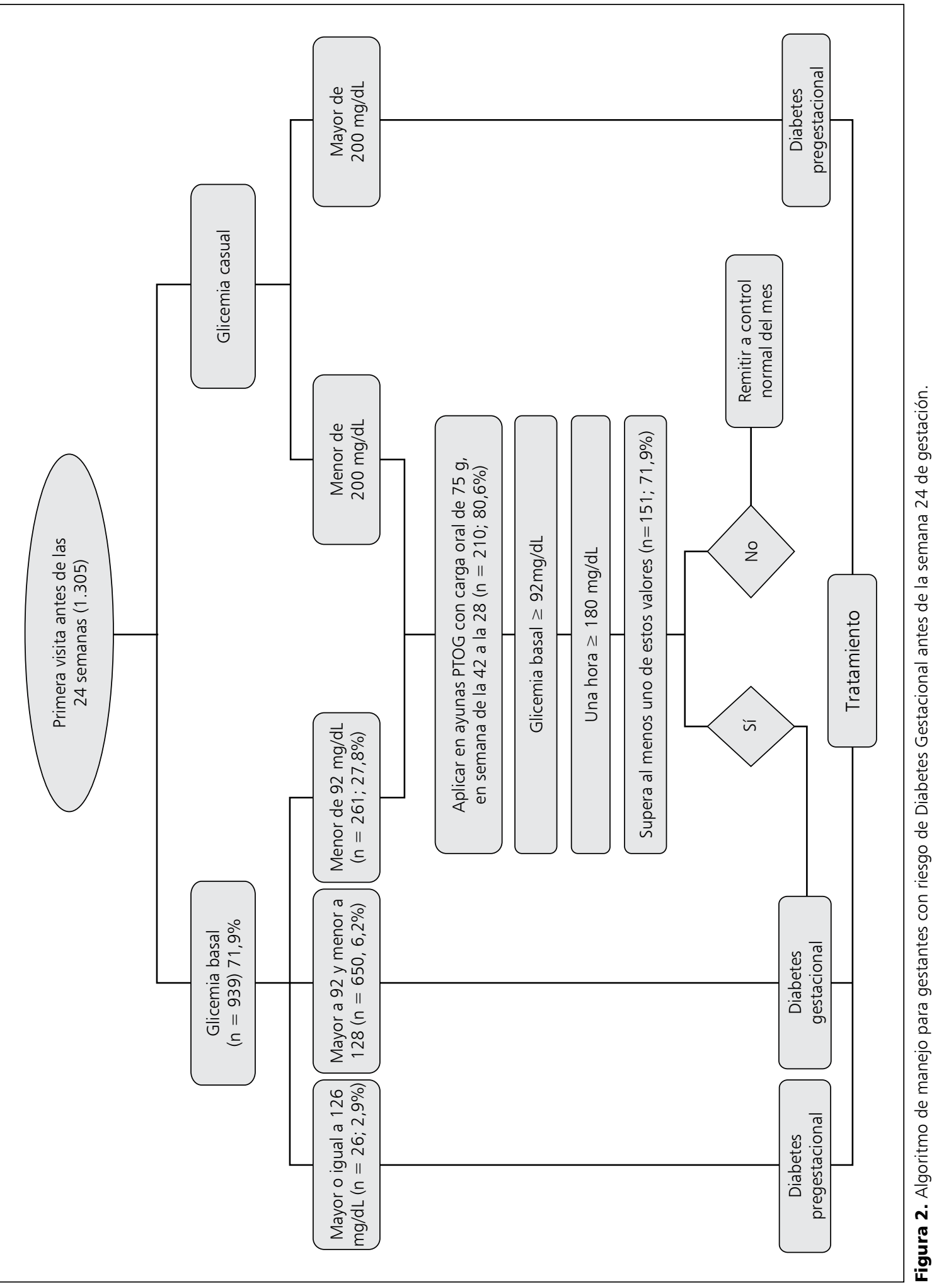




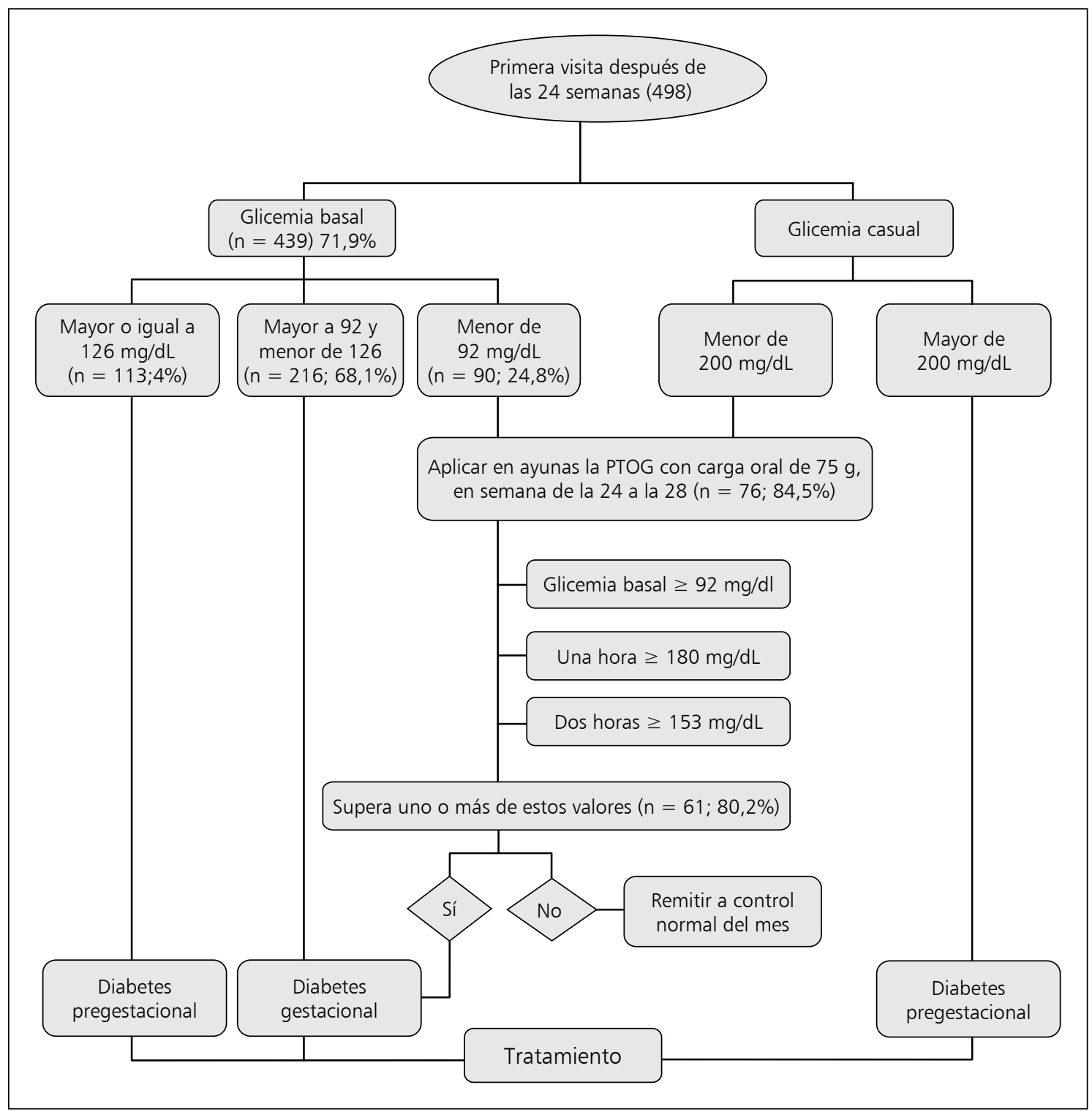

Figura 3. Algoritmo de manejo para gestantes con riesgo de diabetes gestacional después de la semana 24 de gestación.

trimestre o en gestantes sin factores de riesgos descritos, lo que dificulta la detección temprana, si no hay un protocolo específico. Además, si se añade que no hay un consenso actual y adopción universal acerca de la cantidad de gramos que debe contener la carga de glucosa y los diferentes puntos de corte utilizados como criterios diagnósticos para DG, en la PTOG deja entrever retos y desafíos para trabajar en común en la prevención de $\mathrm{DG}^{5,22}$.

Por otra parte, la realización de la PTOG por parte del laboratorio de referencia del ente de salud garantizó el apoyo al programa involucrándose en la fase de actualización de técnicas de laboratorio para detección de DG y estrategias para la implementación de la guía. Esta actuación, acompañada con el adecuado manejo de los centros de atención primaria, retroalimentando al equipo de salud, se traduce en un incremento en los diagnósticos empleando los puntos de corte descritos en el algoritmo utilizando la prueba de tolerancia oral a la 
glucosa con carga de $75 \mathrm{~g}$, mostrando similitudes con lo que registra la literatura ${ }^{2,5,15}$.

La guía de manejo de DG del programa Vida Nueva de la Ciudad de Barranquilla 2012-2014 alcanzó una adherencia de 71,9\% y $63 \%$ para el Grupo 1 y 2 respectivamente; no obstante, es prioritario que esta iniciativa se incorpore a los otros prestadores de la red de salud de tipo privado. Las implementaciones de este tipo de estudios permiten aumentar la evidencia científica para la evaluación de este tipo de guías que, a nivel de Latinoamérica, aunque sí se conocen las guías de manejo, no se ha evaluado el cumplimiento del equipo interdisciplinar para adoptar el protocolo de las gestantes en riesgo; además se desconoce el resultado final y el impacto en detectar eventos de interés que complican la gestación y parto ${ }^{3,4,6}$.

Por otro lado, se puede dimensionar y plantear el grado de organización de la asistencia sanitaria para adoptar e implementar nuevas directrices ${ }^{27}$ además de la trascendencia de sus riesgos. Por lo tanto, es importante conocer y avanzar en las características de las gestantes de tipo demográfico y de morbilidad durante y posterior al embarazo como el impacto financiero y de resultados en salud de gestantes y del uso de servicios, al igual que la adherencia de los lineamientos dentro del sistema de salud que favorezcan una atención de mejor calidad. No obstante preocupa en nuestro país el aumento de sobrepeso y obesidad durante una década 2005-2015 ${ }^{28}$.

Nuestro estudio tiene limitaciones en cuanto que no evaluó desenlaces y comorbilidades a posterior, dado que se documenta a partir de la base de datos disponible y no se registró desenlace y estatus del producto al momento del parto. Por lo tanto, este estudio se limitará a la adopción de la guía a las gestantes que se identificaron sospechosas de riesgo de padecer DG al identificar uno o más factores de riesgos por parte del equipo de médicos que atendieron a las gestantes. En las mujeres con riesgo identificado en el control prenatal se aprecia porcentaje de DG similar a países desarrollados. Dado que esta es una primera aproximación en la detección e identificación de gestantes con DG lo que aporta evidencia para disponer de una línea base de DG en gestantes de escasos recursos que acudan a la red pública del primer nivel de atención. A la fecha se desconoce posibles incrementos en cuidados y atención especializada en gestantes del primer nivel.

\section{Referencias}

1. Varillas C, Blanco S, Gastelu-Iturri J, Reboredo R. Diabetes gestacional: su complejidad y repercusión en la evolución del embarazo y salud del recién nacido. Prog Obstet Ginecol 2005; 48 (6): 289-96.

2. Falavigna M, Pretes I, Schmidt M, Duncan B, Colagiuri $\mathrm{S}$, Roglic G. Impact of gestational diabetes mellitus screening strategies on perinatal outcomes: A simulation study. Diabetes Research and Clinical Practice. 2013; 99: 358-65.

3. Contreras-Zúñiga E, Guillermo-Arango L, Zuluaga-Martínez S, Ocampo V. Diabetes y embarazo. Revista Colombiana de Obstetricia y Ginecología 2008; 59 (1): 38-45.

4. Cascante-Salgado O. Detección de diabetes mellitus gestacional en atención primaria. Revista médica de Costa Rica y Centroamérica 2014; 71 (611): 413-5.

5. Carcoy R, Lumbreras B, Bartha JL, Ricard W. Grupo Español de Diabetes y Embarazo. Gac Sanit 2010; 24 (4): 361-3.

6. López G. Tratamiento de la diabetes en el embarazo: ¿Algo nuevo?. Rev Med Clin Condes 2016; 27 (2): 257 65.

7. Weile L, Khan J, Marseille E, Jensen D, Damm P, Lohse N. Global cost-effectiveness of GDM screening and management: current knowledge and future needs. Best Practice \& research Clinical Obstetrics and Gynecology. 2015; 29: 206-24.

8. Anand S, Gupta M, Teo K, Schulze M, Desai D, Abdala $\mathrm{N}$, et al. Causes and consequences of gestational diabetes in south asians living in canada: results from a prospective cohort study. CMAJ OPEN 2017; 5 (3): E604-11.

9. Xiang AH, Peters RK, Trigo E, et al. Multiple metabolic defects during late pregnancy in women at high risk for type 2 diabetes mellitus. Diabetes 1999; 48: 848-54.

10. O'Sullivan JB. Diabetes mellitus after GDM. Diabetes 1991; 40 Suppl 2: 131-5.

11. Albareda M, Caballero A, Badell G, Piquer S, Ortiz A, de Leiva A, et al. Diabetes and abnormal glucose tolerance in women with previous gestational diabetes. Diabetes Care 2003; 26: 1199-205.

12. Kim C, Newton K, Knopp RH. Gestational diabetes and the incidence of type 2 diabetes. Diabetes Care 2002; 25: 1862-8.

13. Damm P. Diabetes following gestational diabetes mellitus. In: Dornhorst A, Hadden DR, editors. Diabetes and pregnancy. Chichester (UK): Wiley; 1996. p. 341-50.

14. McIntyre H, Colagiuri S, Roglic G. Diagnosis of DDM: A suggested consensus. Best Practice \& research Clinical Obstetrics and Gynecology 2015; 29: 194-205. 
15. Egan A, Vellinga A, Harreiter J, Simmons D, Desoye G, Corcoy R, et al. Epidemiology of gestational diabetes mellitus according to IADPSG/WHO 2013 criteria among obese pregnant women in Europe. Diabetologia 2017; 60 (10): 1913-21.

16. Grupo Español de Diabetes y Embarazo (GEDE) Asistencia a la gestante con diabetes. Guía de práctica clínica actualizada en 2014. Documento de Consenso. Av Diabetol 2015; 31 (2): 45-59.

17. Mayo K, Melamed N, Vandenberghe H, Berger H. The impact of adoption of the international association of diabetes in pregnancy study group criteria for the screening and diagnosis of gestational diabetes. Am J Obstet Gynecol. 2015; 212 (2): 224.e1-9.

18. Lawrence JM, Contreras R, Chen W, Sacks D. Trends in the Prevalence of Preexisting Diabetes and Gestational Diabetes Mellitus Among a Racially/Ethnically Diverse Population of Pregnant Women, 1999-2005. Diabetes Care 2008; 31 (5): 899-904.

19. Concha-López P, Parejo-Linares E, de la Casa-Martí F. Diabetes gestacional: cribado, diagnóstico y seguimiento en el centro de salud. Aten Primaria 2005; 35 (5): 265-8.

20. Khan M, Santana J, Donnellan C, Preston C, Moayyedi P. Medical treatments in the short term management of reflux oesophagitis. Cochrane database Syst Rev 2007; (2): CD003244.

21. Abebe Z, Scifres C, Simhan H, Day N, Catalano P, Bodnar L, et al. Comparisonof Two Screening Strategies for
Gestational Diabetes (GDM2) Trial: Design and rationale. Contemporary Clinical Trials. 2017. doi:10.1016/j. cct.2017.08.012.

22. Massucatti L, Amorim R, Uceli T. Prevalencia de diabetes gestacional en unidades de saude básica. Revista de enfermagem e atencao a saúde 2012; 1 (1): 70-9.

23. Naveiro-Fuentes M, Jiménez-Moleón JJ, Olmedo-Requena N, Amezcua-Prieto C, Bueno-Cabanillas A, Mozas-Romero J. Resultados perinatales en función de 3 criterios diagnósticos de diabetes gestacional. Clin Invest Gin Obst 2015; 42 (2): 66-71.

24. Mendoza H. Detección y manejó de diabetes gestaciónal. WDF-10-572.

25. Lima M, Villalobos M, Aguirre M, Uzcátegui L, Paoli M. Manejo de la diabetes gestacional: Protocolo del Servicio de Endocrinología del Instituto Autónomo Hospital Universitario de los Andes. Rev Venez Endocrinol y Metab 2012; 10 (2): 88-93.

26. Arizmendi J, Carmona V, Colmenares A, Gómez D, Palomo T. Diabetes gestacional y complicaciones neonatales. Rev Fac Med 2012; 20 (2): 50-60.

27. Rubio JA, Ontañon M, Perea V, Megia A, and on behalf of the Spanish Group of Diabetes and Pregnant. Health care of pregnant women with diabetes in Spain: Approach using a questionnaire. Endocrinol Nutr 2016; 63: 113-20.

28. Ministerio de Salud y Protección Social. Encuesta Nacional de Demografía y Salud (ENDS). 2015. Colombia. 